\title{
System Design Model for Versatile Outbreak Surveillance
}

\author{
Hervé Chaudet*1, François Delon², Gaëtan Texier ${ }^{3}$, Jean-Baptiste Meynard², Liliane \\ Pellegrin $^{2}$ and Xavier Deparis ${ }^{2}$
}

'UMR 912 INSERM/Aix-Marseille Université, Marseille, France; ${ }^{2}$ Centre d’Epidémiologie et de Santé Publique des Armées, Marseille,

France; ${ }^{3}$ Institut Pasteur du Cameroun, Yaoundé, Cameroon

\section{Objective}

This paper briefly describes the model for surveillance system design that is used by the ASTER system, which is progressively deployed within the French Forces.

\section{Introduction}

The ASTER system aims at providing an integrated real time epidemiological status of all the French Forces deployed abroad ${ }^{1}$. But, unlike usual surveillance systems, ASTER must cover several target populations exposed to different biological and chemical threats, and the surveillance of each population must be tailored to meet its specific risk profile ${ }^{2}$. Moreover, a surveillance may change at any moment, depending on the evolution of the nature of the threats. For coping with these highly varying surveillance profiles within a same surveillance system, we have developed a formal surveillance system model we have used for designing the collaborations of the system components and allowing the required surveillance versatility.

\section{Methods}

The ASTER formal surveillance model is derived from [3].

The target of the surveillance is a space $\mathrm{E}=<\mathrm{I}, \mathrm{V}>$ where $\mathrm{I}$ is a set of individuals and $\mathrm{V}$ a set of variables. On this space we define a monitoring as a pair $<\mathrm{E}^{\prime}, \mathrm{A}>$ where $\mathrm{E}^{\prime}=<\mathrm{I}^{\prime}, \mathrm{V}^{\prime}, \mathrm{F}>$ is a subspace of $\mathrm{E}$ and $A$ is an aberration detection function with $E^{\prime}$ as input. In $E^{\prime}, V^{\prime}$ is a subset of $\mathrm{V}$, and I' is a subpopulation defined by a selection formula F over V'. Finally, a surveillance is a set of monitorings $S=\left\{M_{1}, M_{2} \ldots\right.$ $M_{n}$ \} over a surveillance space.

Each component of this model finds its counterpart in a surveillance system. The space $\mathrm{E}$ is the surveillance data, each subspace definition $\mathrm{F}$ is a possible epidemiological event, and each $\mathrm{A}$ is a detection algorithm. This model gives a support for building the system knowledge on the basis of its constituents, and the webservice choreography related to the aberration detection on the basis of the relations this model introduces.

\section{Results}

ASTER implements this model using 4 types of webservices.

- Each population under surveillance (i.e. deployment) is a surveillance space $\mathrm{E}$ and a data server exposed within the system as a webservice.

- Each subspace definition $\mathrm{F}$ is a clinical definition or a case detection rule using the signs and symptoms collected on the populations that can be applied over any data server. A webservice keeps all these definition as a library a possible epidemiological event definition.

- Each detection function $\mathrm{A}$ is an aberration detection webservice. This set represents a library of alerting algorithms.

- Finally, a population's surveillance is a set of monitorings defined over a data server, each monitoring combining a clinical definition chosen in the definition library with an alerting algorithm chosen in the algorithm library. Several monitorings may address a same case definition, using different algorithms or different settings of a same algorithm. The system implements surveillances as periodic processes where the results are available by the mean of webservices.

This way of composing surveillances by combining populations, epidemiological events and aberration detections from libraries gives the system a significant degree of flexibility. We have introduced in ASTER the possibility to keep pre-defined surveillances in a specific library, ready to be activated at any moment for dealing with some possible special health circumstances.

\section{Conclusions}

This surveillance model enables a same system to provide a unified monitoring of several populations exposed to different health threats and to quickly respond to a threat evolution. The system currently covers populations in desertic areas as well as in Amazonian Forest. This versatility allowed a quick and easy system tailoring for the recent French Deployments in Jordanian Refugees Camps or in Mali.

\section{Keywords}

surveillance model; system design; surveillance library; military surveillance system; ASTER

\section{References}

[1] Chaudet H, Pellegrin L et al. Web services based syndromic surveillance for early warning within French Forces. Stud Health Technol Inform. 2006;124:666-71.

[2] Meynard JB, Chaudet $\mathrm{H}$ et al. Advantages and limits of real-time epidemiological surveillance during military deployments: the experience of the French Armed Forces. Mil Med. 2009 Oct;174(10):1068-74.

[3] Albusac J, Vallejo D et al. Intelligent surveillance based on normality analysis to detect abnormal behaviors. IJPRAI 2009;23(7):1223-1244.

\section{*Hervé Chaudet}

E-mail: herve.chaudet@univ-amu.fr 